\title{
The origin of UHECR: the distance to the nearest source and the dipole
}

\section{Rodrigo Guedes Lang, ${ }^{a, *}$ Andrew M. Taylor ${ }^{b}$ and Vitor de Souza ${ }^{c}$}

${ }^{a}$ Friedrich-Alexander-Universität Erlangen-Nürnberg, Erlangen Centre for Astroparticle Physics, Erwin-Rommel-Str. 1, D 91058 Erlangen, Germany

${ }^{b}$ DESY, D-15738 Zeuthen, Germany

${ }^{c}$ Instituto de Física de São Carlos, Universidade de São Paulo, Av. Trabalhador São-Carlense, 400, São Carlos, SP, Brazil

E-mail: rodrigo. lang@fau.de

\begin{abstract}
A semi-analytical, fast and robust method for the propagation of ultra-high-energy cosmic rays (UHECR) under extra-galactic turbulent magnetic fields presented by the authors in previous works is described in detail. Under the assumption of dominant turbulent fields, it is possible to obtain the energy spectrum, as well as the relative contribution from each source, and the angular distribution of arrival directions without the need for 4-D simulations. This method provides an important tool in the quest for the origin of UHECR. A brief overview of the results already obtained with it is presented. The relative contribution to the energy spectrum from sources in different distance shells is elucidated and constraints of a few tens of Mpc, depending on composition, are imposed to the distance to the nearest source. A solid understanding of the arising of the dipole as well as its evolution with energy is built. The importance of a power spectrum analysis in energies detected by experiments is discussed.
\end{abstract}

$37^{\text {th }}$ International Cosmic Ray Conference (ICRC 2021)

July 12th - 23rd, 2021

Online - Berlin, Germany

\footnotetext{
*Presenter
} 


\section{Introduction}

The origin of ultra-high-energy cosmic rays (UHECR, $E>10^{18} \mathrm{eV}$ ) is one of the current key topics in astroparticle physics, being an open question for many decades [1]. These particles are the most energetic events ever detected and could, thus, be intrinsically related to extreme environments of the Universe. Nevertheless, these particles are charged and consequently deviated by galactic and extra-galactic magnetic fields (GMF and EGMF, respectively), not pointing directly to their sources. Information about their origin, however, is not completely lost during propagation and the distribution of arrival directions could still be used to elucidate about their sources.

Over the last two decades, two dedicated observatories covering large areas on the ground have been detecting UHECR, the Pierre Auger Observatory [2] in the southern hemisphere and the Telescope Array [3] in the northern hemisphere. Deviations from isotropy in arrival directions have been measured, from which we highlight the large-scale anisotropy measured by the Pierre Auger Observatory $[4,5]$. A dipole of $6.5 \%$ for UHECR with $E>8 \mathrm{EeV}$ pointing outwards the galactic center was detected with $5.2 \sigma$ confidence level. The evolution with energy of the amplitude and phase of the dipole in right-ascension has also been obtained, which shows a shift of the phase from near the galactic center up to $\sim 1 \mathrm{EeV}$ to outwards the galactic center for energies above $8 \mathrm{EeV}$. These are strong indications of a change from a predominance of galactic sources to a predominance of extra-galactic sources around a few EeV.

Several works have tried to model the arising of the dipole and its evolution with energy [610]. These models usually rely on Monte Carlo simulations for the propagation of UHECR. The computational cost of such simulations increases substantially when magnetic fields are considered since a 4-D approach is needed, i.e., the simulations need to keep track of the 3 spatial directions as well as the time dimension of each cosmic ray. The fraction of simulation events that do not arrive on Earth also becomes quite large, leading to a significant worsening of statistics. If only turbulent fields are considered, however, symmetry arguments can be used to simplify the problem such that it can be treated with 1-D simulations again. Using that, refs. [11] and [10] proposed a semi-analytical approach for the propagation, allowing a robust and faster calculation of the energy spectrum, composition, and distribution of arrival directions. In this proceeding, we describe in more detail this method, which is presented in section 2 . We also provide a brief overview of the astrophysical insights obtained using them in section 3. Conclusions are addressed in section 4 .

\section{Semi-analytical method for the propagation of UHECR in turbulent magnetic fields}

When only turbulent fields are considered, the propagation of cosmic rays emitted by a single source becomes radially symmetric. Therefore, neglecting the effects of structured fields allows for a simplification of the treatment of the propagation. In this work, only turbulent EGMF are considered. We appeal to the results of quasi-linear theory [12] to use a simple prescription for them, in which UHECR have their direction of movement completely randomized after traveling a given distance following a Poisson distribution, $e^{-x / \lambda_{\text {scatt }}} . \lambda_{\text {scatt }}$ is the scattering length and is given by 


$$
\lambda_{\text {scatt }}=\lambda_{\mathrm{coh}}\left\{\begin{array}{ll}
\left(\frac{R_{L}}{\lambda_{\mathrm{coh}}}\right)^{1 / 3}, & \text { for } R_{L}<\lambda_{\mathrm{coh}} \\
\left(\frac{R_{L}}{\lambda_{\mathrm{coh}}}\right)^{2}, & \text { for } R_{L} \geq \lambda_{\mathrm{coh}}
\end{array},\right.
$$

where $\lambda_{\text {coh }}$ is the coherence length of the field and $R_{L}$ is the Larmor radius of the particle, which depends on the intensity of the field, $B$, and the particle energy, $E$, and charge, $Z$, as

$$
R_{L}=\frac{p}{|q| B} \approx \frac{1.081}{Z}\left(\frac{E}{\mathrm{EeV}}\right)\left(\frac{\mathrm{nG}}{B}\right) \mathrm{Mpc}
$$

\subsection{Radial distribution}

Since radial symmetry is expected in the propagation in this case, it is important to look at the radial distribution of cosmic rays emitted by a single source.

Initially, we consider a pulse of cosmic rays emitted in a short time interval such that $d N / d t=$ $1 / \tau$. Three main propagation regimes are found. For short times (in comparison with $\lambda_{\text {scatt }} / c$ ), the propagation is mostly ballistic, i.e., the effects of the magnetic fields are negligible. For long times, the propagation is mostly diffusive. An intermediate regime is also found.

For the short time regime, we describe the radial distribution using a delta function,

$$
\left.\frac{d^{2} N}{d r d t}\right|_{c t / \lambda_{\text {scatt }} \rightarrow 0}=\frac{\delta(r-c t)}{\tau} .
$$

For the long time regime, a truncated Gaussian is used,

$$
\left.\frac{d^{2} N}{d r d t}\right|_{c t / \lambda_{\text {scatt }} \rightarrow \infty}=\left\{\begin{array}{ll}
\tau^{-1} A r^{2} e^{-\frac{r^{2}}{2 \sigma^{2}}} & \text { for } r \leq c t \\
0 & \text { for } r>c t
\end{array},\right.
$$

with $\sigma=\lambda_{\text {scatt }} c t / 3$. The truncation is needed to avoid supraluminal propagation and leads to the following normalization

$$
\frac{1}{A}=\sigma^{2}\left(\sqrt{\frac{\pi}{2}} \sigma \operatorname{erf}\left(\frac{c t}{2 \sigma}\right)-c t e^{-\frac{(c t)^{2}}{2 \sigma^{2}}}\right) .
$$

Nevertheless, a more complex distribution is needed in the transition regime, for which we use a Jüttner distribution [13], given by

$$
\frac{d^{2} N}{d r d t}=\left\{\begin{array}{ll}
\tau^{-1} \frac{r^{2} \alpha e^{-\alpha / \sqrt{1-\left(\frac{r}{c t}\right)^{2}}}}{(c t)^{3} K_{1}(\alpha)\left(1-\left(\frac{r}{c t}\right)^{2}\right)^{2}} & \text { for } r \leq c t \\
0 & \text { for } r>c t
\end{array},\right.
$$

with $\alpha=3 c t / \lambda_{\text {scatt }}$ and where $K_{1}$ is the modified Bessel function of the second kind. The Jüttner distribution is more complete and can be reduced to a delta or a Gaussian distribution if the right limits are taken. It is, however, much more complex to treat analytically and to solve numerically. Therefore, a trade-off between precision and feasibility is found by setting the three regimes as: ballistic (delta distribution) for $\alpha<0.1$, transition (Jüttner distribution) for $0.1<\alpha<10$, and diffusive (truncated Gaussian distribution) for $\alpha>10$. 
The radial distribution of a source emitting over time, instead of a single pulse, can finally be obtained by integrating $d^{2} N / d r d t$ over time. While there might be time-dependent emissivities, $\tau^{-1}(t)$, it is common to assume the sources as standard candles emitting constantly over time. The upper interval of the integration is the age of the sources, which is approximated as the Hubble time.

We consider that the scattering length of cosmic rays does not change much during propagation. This is a reasonable assumption, since the main energy loss, i.e., the photodisintegration will lead to a change of only $\approx 0.5$ in the rigidity in the worst case. The electron pair production, which dominates in the case of protons, has already been shown to be safely neglected in this context [14].

\subsection{Energy spectrum}

One of the main reasons for using Monte Carlo simulations to calculate the propagation of UHECR is to treat the energy losses, in particular, the photodisintegration, which changes the number and species of the cosmic rays. These energy losses, however, depend only on the total distance traveled by the cosmic ray (or, correspondingly, the time traveled), i.e., a cosmic ray traveling a given distance in a straight line will lose on average the same energy as a cosmic ray scattering over multiple directions but traveling the same total distance. For that reason, we simulated 1-D propagation in the CRPropa3 code [15] for different travel times. A 4-D mapping can be done to obtain the energy spectrum of cosmic rays arriving from each source by convoluting the simulated spectrum with the radial distributions discussed in the previous section.

\subsection{Arrival directions}

Ref. [11] also proposes an extension of the method for obtaining the distribution of arrival directions as well as its harmonic coefficients based on the radial distributions previously discussed. In order for a cosmic ray to arrive on Earth with an angle $\theta$ between the source and the arrival direction, it must have last scattered somewhere in the line of sight described by that angle and traveled all the way to Earth without scattering again. One can, thus, obtain the total number of cosmic rays arriving in that direction by integrating over the line of sight the cosmic rays which fulfill these criteria. The density of cosmic ray in any point $l$ on that line is given by $d N / d r(r)$, with $r$ written as

$$
r^{2}=l^{2}+r_{s}^{2}-2 l r_{s} \cos \theta
$$

where $\theta$ is the angle between the source position and the given direction from Earth and $r_{s}$ is the distance from Earth to the source.

The fraction of cosmic rays which scatter over a time interval $d t$ is $c d t / \lambda_{\text {scatt }}$ and the fraction of cosmic rays which travel from that point to Earth without scattering again is $e^{-l / \lambda_{\text {scatt }}}$. With that, the density of cosmic rays arriving in the direction $\theta$ in a given interval $d t$ is calculated by

$$
\left.\frac{d^{2} N}{d A d t}\right|_{\text {scatt }}(\theta, t)=\int_{0}^{\infty} \frac{d l e^{-l / \lambda_{\text {scatt }}}}{\lambda_{\text {scatt }} / c} \int_{t+l} \frac{d t^{\prime}}{\tau} \frac{d^{3} N}{d r^{3}}\left(r, t^{\prime}\right) .
$$

Nevertheless, another contribution needs to be taken into account. Some cosmic rays will travel ballistically all the way from the source without ever scattering. These events will point to the direction of the source $(\theta=0)$ and their density can be obtained by 


$$
\left.\frac{d^{2} N}{d A d t}\right|_{\text {ballist }}(\theta, t)=\delta(\theta=0) \frac{e^{-r_{s} / \lambda_{\text {scatt }}}}{\tau 4 \pi r_{s}^{2}},
$$

in which $\delta(\theta=0)$ guarantees that the events point in the right direction. Finally, the total angular distribution of arrival directions will be given by

$$
\frac{d^{2} N}{d A d t}=\left.\frac{d^{2} N}{d A d t}\right|_{\text {scatt }}+\left.\frac{d^{2} N}{d A d t}\right|_{\text {ballist }} .
$$

\subsection{Spherical harmonics expansion}

It is common to analyze the distribution of arrival directions via its spherical harmonics. The angular distribution can be expanded as

$$
\frac{d N}{d \cos \theta}=N_{s}\left[1+\sum_{n=1}^{\infty} \sum_{m=-\ell}^{m=\ell} \Phi_{\ell, m} Y_{\ell}^{m}(\phi, \theta)\right] .
$$

For the case of a single source and the approximation of turbulent fields here considered, the symmetry implies no dependency on $\phi$ and, thus, only the terms for $m=0$ are considered. With that, the spherical harmonics are defined as just renormalized Legendre polynomials [16]

$$
Y_{\ell}(\theta)=Y_{\ell}^{0}(\theta)=\frac{2 \ell+1}{\sqrt{2}} P_{\ell}(\cos \theta)
$$

and the coefficients $\Phi_{\ell}$ are obtained by

$$
\Phi_{\ell}=\int_{-1}^{1} d \cos \theta \frac{d N}{N d \cos \theta} \frac{2 \ell+1}{\sqrt{2}} P_{\ell}(\cos \theta)
$$

with $\Phi_{1}$ being the dipole. Important pieces of information about the distribution are found by looking at its angular power spectrum, which gives the importance of each multipole in the spherical harmonics expansion. The coefficients of the power spectrum are given by

$$
C_{\ell}=\frac{\Phi_{\ell}^{2} /(2 \ell+1)}{\sum_{n} \Phi_{n}^{2} /(2 n+1)},
$$

with

$$
\begin{aligned}
& 0<C_{\ell}<1, \text { for all } \ell \\
& \sum_{\ell=0}^{\infty} C_{\ell}=1 .
\end{aligned}
$$

The angular distribution as a function of the energy for an ensemble of sources, $d N / d \cos \theta(E)$, can then be obtained by summing the distribution from each source, $s$, normalized by its respective contribution to the total flux, $N_{s}(E) / N_{\text {tot }}(E)$. Such contribution can be calculated using the radial distributions previously discussed. The simple case of sources randomly distributed is discussed in detail in Ref. [10]. 
When an ensemble with a large number of sources is considered, the higher orders multipoles usually vanish, with only $C_{0}$ and maybe $C_{1}$ being significant. However, for the highest energies detected by experiments and some realistic combination of hypotheses (such as composition, EGMF intensity, and source density), that is not the case. In order to understand when these must be taken into account, we define the non-dipolarity term, $f_{\text {non-dip }}=1-C_{0}-C_{1}$, as a quantification of the importance of the higher-order multipoles.

\section{Overview of the physical insights obtained}

With the semi-analytical method described above, Ref. [11] has studied the effects of the magnetic fields and distance to the nearest source on the energy spectrum, while Ref. [10] has studied the effect of several astrophysical hypotheses on the evolution of the dipole and power spectrum with energy. In this proceeding, we provide a brief compilation of the most important results obtained in these two works.

A good understanding of the effects of magnetic fields and distance of the nearest source is obtained by looking at the contribution to the spectrum from sources divided into different distance shells. Up to five regimes are found, from highest to lowest energy:

- A. Ballistic propagation: for the highest energies, the effects of the magnetic fields are negligible and, consequently, the propagation is ballistic. This regime can be divided into two subregimes:

- AI. Energy-loss horizon: a strong horizon appears due to energy-dependent energy losses;

- AII. Unimpeded ballistic propagation: depending on the EGMF considered, there may be an energy range in which most of the propagation is still ballistic but the energy losses are not so dominant;

- B. Diffusive propagation: for the energy ranges where the effects of magnetic fields are dominant, three subregimes are found:

- BI. Non-resonant scattering enhancement: the contribution to the flux is increased due to cosmic rays scattering over time and being accumulated. The enhancement grows as $E^{-2}$, following the EMGF prescription from eq. 1;

- BII. Resonant scattering enhancement: same as the previous one, but with the enhancement growing as $E^{-1 / 3}$, following the EMGF prescription from eq. 1;

- BIII. Magnetic horizon: a drop in the contribution is seen due to the finite age of the Universe.

The energies in which the transitions between the regimes appear are different for different distance shells, happening at higher energies for farther sources. This leads to three main regimes when looking at the total spectrum: at the highest and lowest energies the spectrum is dominated by local sources, while in the intermediate energies the spectrum is dominated by the farther ones. Considering a distance to the nearest source (and thus decreasing the number of local sources) 
leads to two horizons, one at each end of the spectrum. Ref. [11] used these effects to constrain the maximum distance to the nearest source, showing that sources at maximum a few tens of Mpc, depending on composition, are essential to describe the energy spectrum measured by the Pierre Auger Observatory.

A solid understanding of the arising of the dipolar behavior as well as how it evolves with energy could also be built by looking at the contribution from sources in different distance shells [11, 17]. The regimes just discussed dictate the evolution of the dipole with energy. It is also shown that the dipole is driven by the nearest sources but diluted by farther ones. This means that its direction will be dictated by the local sources while its amplitude will strongly depend on the source density, and, correspondingly, the number of contributing sources.

\section{Conclusions}

In this proceeding, we have discussed the semi-analytical method for calculating both the energy spectrum and distribution of arrival directions for UHECR propagating in environments with EGMF.

The method allows for a much faster while still robust simulation procedure by reducing from four dimensions to one under the assumption that the fields are turbulent and not structured. An analytical mapping is used, convoluting the radial distribution of scattering cosmic rays. With that, one can obtain the energy spectrum and arriving composition as well as relative contributions from different sources at different distances.

The possibility of calculating the angular distribution of arrival directions integrating the radial distributions is also presented. From that, a quantitative analysis via spherical harmonics can be done.

While the origin of UHECR still remains an unsolved puzzle, important experimental results have shed light on this question over the last years. It is important to develop theoretical and phenomenological models to describe such measurements. Such models, however, are limited by several unknowns about the astrophysics involved and by computational difficulties. This work joins these efforts by providing a robust tool for studying different models and assumptions with a decent computational cost and the possibility of testing a wide range of hypotheses.

\section{Acknowledgements}

VdS acknowledges FAPESP support No. 2015/15897-1. VdS acknowledge the National Laboratory for Scientific Computing (LNCC/MCTI, Brazil) for providing HPC resources of the SDumont supercomputer, which have contributed to the research results reported within this paper. (http: //sdumont. Incc. br). VdS thanks CNPq.

\section{References}

[1] R. Alves Batista et al., Open Questions in Cosmic-Ray Research at Ultrahigh Energies, Front. Astron. Space Sci. 6 (2019) 23 [1903.06714]. 
[2] Pierre Auger collaboration, The Pierre Auger Cosmic Ray Observatory, Nucl. Instrum. Meth. A 798 (2015) 172 [1502 . 01323].

[3] H. Kawai, S. Yoshida, H. Yoshii, K. Tanaka, F. Cohen, M. Fukushima et al., Telescope Array experiment, Nuclear Physics B: proceedings supplements 175-176 (2008) 221 .

[4] Pierre Auger collaboration, Observation of a Large-scale Anisotropy in the Arrival Directions of Cosmic Rays above $8 \times 10^{18} \mathrm{eV}$, Science 357 (2017) 1266 [1709.07321].

[5] PIERre Auger collaboration, Cosmic-ray anisotropies in right ascension measured by the Pierre Auger Observatory, Astrophys. J. 891 (2020) 142 [2002.06172].

[6] D. Harari, S. Mollerach and E. Roulet, Anisotropies of ultrahigh energy cosmic ray nuclei diffusing from extragalactic sources, Phys. Rev. D 92 (2015) 063014 [1507.06585].

[7] N. Globus and T. Piran, The extragalactic ultra-high energy cosmic-ray dipole, Astrophys. J. Lett. 850 (2017) L25 [1709. 10110].

[8] A. Dundović and G. Sigl, Anisotropies of Ultra-high Energy Cosmic Rays Dominated by a Single Source in the Presence of Deflections, JCAP 01 (2019) 018 [1710.05517].

[9] S. Mollerach and E. Roulet, Extragalactic cosmic rays diffusing from two populations of sources, Phys. Rev. D 101 (2020) 103024 [2004.04253].

[10] R.G. Lang, A.M. Taylor and V. de Souza, Ultrahigh-energy cosmic rays dipole and beyond, Phys. Rev. D 103 (2021) 063005.

[11] R.G. Lang, A.M. Taylor, M. Ahlers and V. de Souza, Revisiting the distance to the nearest ultrahigh energy cosmic ray source: Effects of extragalactic magnetic fields, Phys. Rev. D 102 (2020) 063012.

[12] R. Schlickeiser, Cosmic-Ray Transport and Acceleration. I. Derivation of the Kinetic Equation and Application to Cosmic Rays in Static Cold Media, 336 (1989) 243.

[13] R. Aloisio, V. Berezinsky and A. Gazizov, Superluminal problem in diffusion of relativistic particles and its phenomenological solution, Astrophys. J. 693 (2009) 1275 [0805 . 1867].

[14] S. Mollerach and E. Roulet, Magnetic diffusion effects on the ultra-high energy cosmic ray spectrum and composition, JCAP 10 (2013) 013 [1305. 6519].

[15] R. Alves Batista et al., CRPropa 3 - a Public Astrophysical Simulation Framework for Propagating Extraterrestrial Ultra-High Energy Particles, JCAP 05 (2016) 038 [1603.07142].

[16] D. Harari, S. Mollerach and E. Roulet, Anisotropies of ultrahigh energy cosmic rays diffusing from extragalactic sources, Phys. Rev. D 89 (2014) 123001 [1312 . 1366].

[17] A.M. Taylor, M. Ahlers and F.A. Aharonian, The need for a local source of UHE CR nuclei, Phys. Rev. D 84 (2011) 105007 [1107. 2055]. 